Open Peer Review on Qeios

\title{
Diagnostic accuracy of 'modified' Pediatric Sleep Questionnaire (PSQ) for Obstructive Apnea Syndromes in pediatric age
}

Paola Spinuzza $^{1}$, Giovanna Giuliana ${ }^{1}$, Laura Maniscalco ${ }^{1}$, Vincenza Manzella ${ }^{1}$, Giuseppe Pizzo ${ }^{1}$, Domenica Matranga $^{1}$, Gaetano La Mantia ${ }^{1}$, Vera Panzarella ${ }^{1}$

1 University of Palermo

Funding: The author(s) received no specific funding for this work.

Potential competing interests: The author(s) declared that no potential competing interests exist.

\section{Abstract}

Obstructive sleep apnoea syndrome (OSAS) is a sleep-related breathing disorders (SRBDs) characterized by total or partial collapse of the upper airways during sleep. The Paediatric Sleep Questionnaire (PSQ) is one of the most used and validated screening tools, but it lacks the comprehensive assessment of some determinants of OSAS, specifically anamnestic assessment and sleep quality. This lack with modified PSQ was addressed in a recent study which however had some limitations such as a small sample. This study aims to overcome these limitations by involving a larger sample. The modified PSQ was administered via a digital form to the parents of children (age range: 3-18 years) attending schools in Palermo (Sicily-Italy). For each item, added in the original PSQ, sensitivity and specificity, positive and negative predictive values, and positive and negative likelihood ratios were calculated. The highest sensitivity $(71.4,95 \% \mathrm{Cl}: 29,0 ; 96,3)$, in combination with the highest specificity $(60.0,95 \%$ IC:

$51.9 ; 67.6)$, was found for the Item 8 ('Does your child sweat at night?'). Furthermore the highest specificity $(96.895 \% \mathrm{Cl}: 92.8 ; 98.9)$, in combination with the highest sensitivity $(14.2,95 \% \mathrm{Cl}: 0.3 ; 57.8)$ was found for the Item 11 ('Does your child have confused awakenings, e.g., the child looks awake but confused, disoriented and sometimes aggressive?'). Compared to the previous study, this study highlights how other Items related to sleep quality can be essential for the diagnosis of OSAS. Although these results should be validated with clinical diagnoses, they suggest that including the factors discriminating sleep quality could further increase the efficiency and accuracy of PSQ.

Keywords: paediatric Sleep-Related Breathing Disorders (SRBD); paediatric Obstructive Sleep Apnoea Syndrome (OSAS); Paediatric Sleep Question (PSQ); Di.Chir.On.S 2021

\section{Introduction}

Respiratory sleep disorders, and mainly OSAS, are frequent in subjects of developmental age. In the 
paediatric population, it is especially important to diagnose them early in order to reduce the impact of further related comorbidities (e.g., obesity or excess weight, hypertension, and cardiovascular and metabolic diseases [1][2]. To this end, various screening questionnaires have been discussed in the literature, such as SCR, OSA-18 [3], BS, “I'm sleep" SSSDR and PSQ [4]. Among these, PSQ would seem to be the best screening tool, with the highest sensitivity and specificity (equal to 78\% and 72\%)[1][5][6][7][8]. However, according to a recent literature search, PSQ does not evaluate some determinants related to the anamnestic evaluation and the sleep quality that are decisive for the diagnosis of OSA.

In this regard, a recent study ${ }^{[9]}$ assessed the accuracy of some specific items added to the original PSQ, particularly related to the patient's anamnestic history and to the quality of sleep, for the screening of OSAS in a paediatric population living in Sicily (Italy). The "anamnestic" dimension included 7 items investigating: ethnicity (Item1); whether the child was born premature or full-term (Item 2); the presence of previous illnesses (Item 3); familiarity with sleep breathing problems (Item 4); the presence of seasonal allergies, asthma or nasal congestion (Item 5); the presence of respiratory diseases(Item 6); and the presence or absence of adenoids (Item 7). The "sleep quality" dimension included 8 items investigating particular behaviours of the child during the night that are considered useful for assessing the quality of sleep: the tendency to sweat (Item 8); and frequent arousal (Item 9); the assumption of bizarre positions (Item 10); confused awakenings (Item 11); sleepwalking (Item 12); the tendency to drool during sleep (Item 13); nightmares (Item 14); and, finally, insomnia (Item 15). All the selected items are detailed in Table 1. In this recent study the assumption of bizarre or abnormal positions during sleep (Item 10) was found statistically significant for predicting the occurrence of OSAS in children ( $p$-value $\leq 0.003$ ). However, the main limitation of the previous study is the small sample of investigated population; so, this investigation aims to involve a larger sample with a greater age range.

\section{Materials and Methods}

The modified PSQ, with the 15 added items, was administered from 6 May to 26 June 2021 to a population of parents of children in developmental age (age range: 3-18 years) of public schools located in Palermo (Sicily, Italy). The questionnaire was administered via digital form (Google form) and, differently from the previous study [9], was extended to a larger population sample, thanks the use of other computer application systems (e.g WhatsApp, Facebook). In this way it could be widely and easily distributed in the COVID-19 pandemic period. Before filling out the questionnaire, each parent had to give consent for data processing in compliance with Italian and European Union policies on the management of sensitive data. Male or female children not presenting syndromic disorders (i.e., achondroplasia; Beckwith-Wiedemann syndrome; Down syndrome; Ehlers-Danlos syndrome; Ellis-van Creveld syndrome; Noonan syndrome; Pierre Robin sequence/complex; Prader-Willi syndrome; sickle cell diseases) were considered eligible for inclusion. In the case of more than one eligible child within the same family unit, the parents were asked to complete the questionnaire for each of their children. For each of 15 items, added from the modified PSQ, sensitivity and specificity, positive and negative predictive values, and positive and negative likelihood 
ratios were calculated via MDCalc statistical software. For the diagnosis of OSAS, the optimal cut-off of 0.33 was used, as suggested by Chervin et al. $2000^{[6]}$.

\section{Results}

One hundred and sixty-seven modified PSQs were completed correctly. Of the 167 children, the average age was 7,7 $\pm 2,78$ (SD) and the range was $3-18$ years. There were $86(51,5 \%)$ males with an average age of $7,7 \pm 3.68$ (SD) and $82(48,5 \%)$ females with an average age of 7,8 3,40 (SD). Relating to the evaluation of the additional items, the highest sensitivity $(71.4,95 \% \mathrm{Cl}: 29,0 ; 96,3)$, in combination with the highest specificity $(60.0,95 \%$ IC: $51.9 ; 67.6)$, was found for the Item 8 ('Does your child sweat at night?'). Furthermore the highest specificity $(96.895 \% \mathrm{Cl}: 92.8 ; 98.9)$, in combination with the highest sensitivity $(14.2,95 \% \mathrm{Cl}$ : 0.3;57.8) was found for the Item 11 ('Does your child have confused awakenings, e.g., the child looks awake but confused, disoriented and sometimes aggressive?').

Results are shown in Tables 2 and 3.

\section{Conclusions}

Compared to the previous study, this analysis highlights how other Items related to sleep quality can be essential for the diagnosis of OSAS. In addition to item 10, whose high sensitivity and specificity are confirmed by this study, items 8 and 11 were also statistically significant. However the item 8 appears to be of dubious interpretation, since the questionnaire was administered in a summer month, therefore excessive sweating could also be due to other factors not related to OSA. Item 11 shows how excessive sweating associated with shortness of breath during sleep and the assumption of abnormal or bizarre positions by the child to reduce perceived obstruction in the upper airways are common.

Although these results should be validated with clinical/instrumental diagnoses, they support that a simple and careful observation of the child during sleep by the parents is essential to better screen subjects with suspected OSAS. 


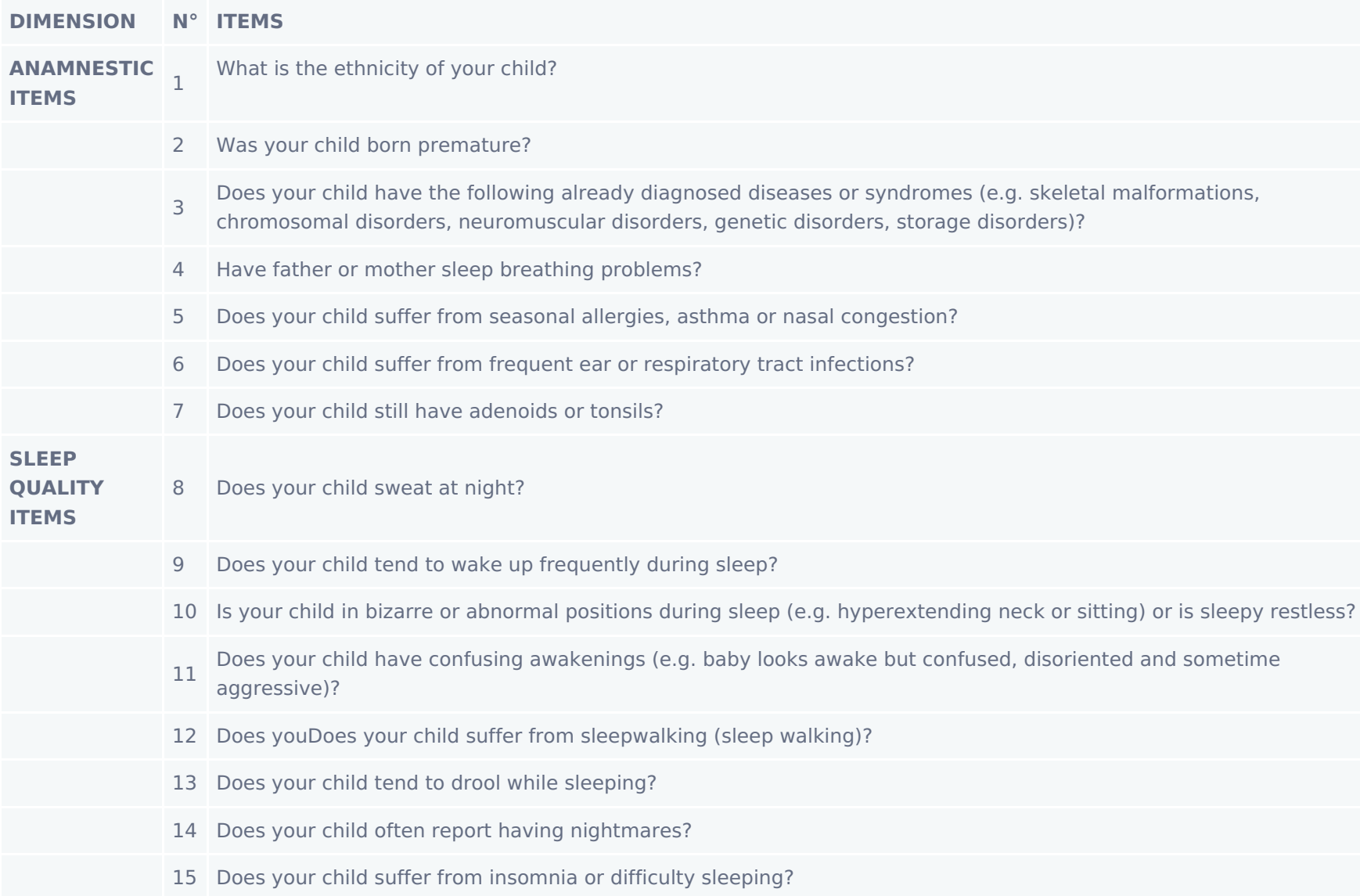

Tab2. Accuracy of the additional 7 items (anamnestic items) for OSAS: sensitivity, specificity, predictive values and likelihood-ratios (with 95\%Cls).

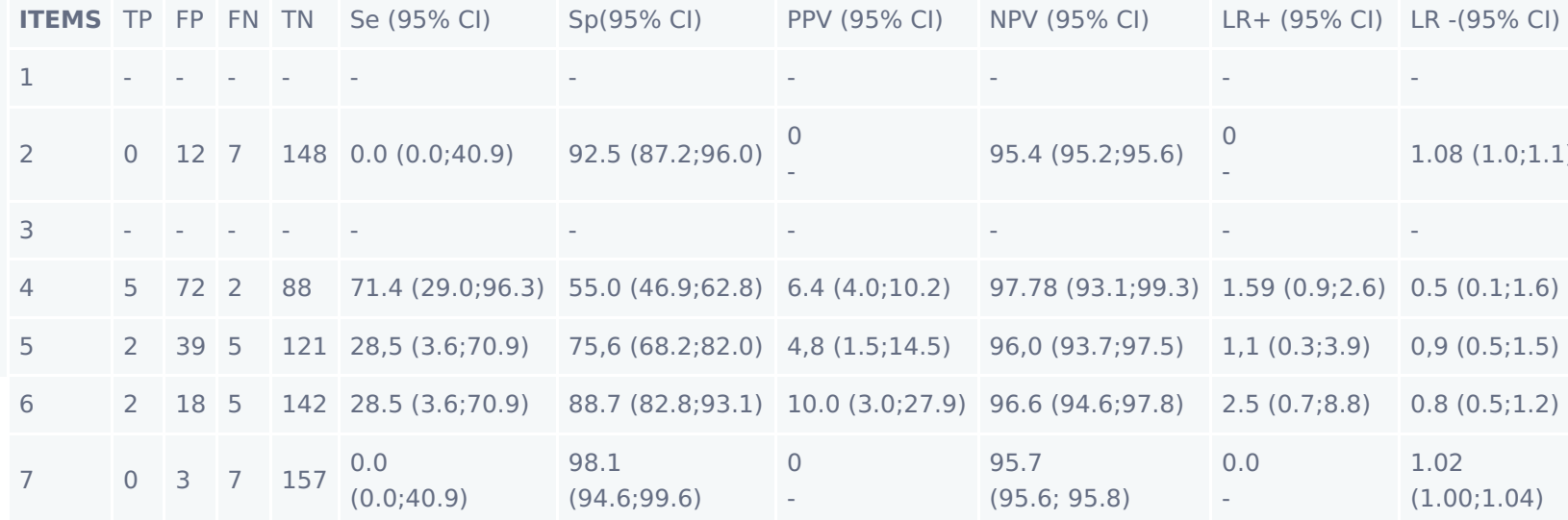

Tab 3. Accuracy of the additional 8 items (sleep quality items) for OSAS: sensitivity, specificity, predictive values and likelihood-ratios (with 95\%Cls). 


\begin{tabular}{|c|c|c|c|c|c|c|c|c|c|c|}
\hline ITEMS & TP & FP & $\mathrm{FN}$ & TN & Se $(95 \% \mathrm{Cl})$ & $\mathrm{Sp}(95 \% \mathrm{Cl})$ & PPV $(95 \% \mathrm{Cl})$ & NPV $(95 \% \mathrm{Cl})$ & $\mathrm{LR}+(95 \% \mathrm{Cl})$ & LR- $(95 \% \mathrm{Cl})$ \\
\hline 8 & 5 & 64 & 2 & 96 & $\begin{array}{l}71.4 \\
29.0 ; 96.3\end{array}$ & $\begin{array}{l}60.0 \\
(51.9 ; 67.6)\end{array}$ & $\begin{array}{l}7.2 \\
(4.5 ; 11.4)\end{array}$ & $\begin{array}{l}97.9 \\
(93.6 ; 99.3)\end{array}$ & $\begin{array}{l}1.7 \\
(1.0 ; 2.9)\end{array}$ & $\begin{array}{l}0.4 \\
(0.1 ; 1.5)\end{array}$ \\
\hline 9 & 1 & 10 & 6 & 150 & $\begin{array}{l}14.2 \\
(0.3 ; 57.8)\end{array}$ & $\begin{array}{l}93.7 \\
(88.8 ; 96.9)\end{array}$ & $\begin{array}{l}9.0 \\
(1.4 ; 40.3)\end{array}$ & $\begin{array}{l}96.1 \\
(94.8 ; 97.1)\end{array}$ & $\begin{array}{l}2.2 \\
(0.3 ; 15.4)\end{array}$ & $\begin{array}{l}0.9 \\
(0.6 ; 1.2)\end{array}$ \\
\hline 10 & 2 & 11 & 5 & 149 & $\begin{array}{l}28.5 \\
(3.6 ; 70.9)\end{array}$ & $\begin{array}{l}93.1 \\
(88.0 ; 96.5)\end{array}$ & $\begin{array}{l}15.3 \\
(4.7 ; 40.0)\end{array}$ & $\begin{array}{l}96.7 \\
(94.9 ; 97.9)\end{array}$ & $\begin{array}{l}4.1 \\
(1.1 ; 15.2)\end{array}$ & $\begin{array}{l}0.7 \\
(0.4 ; 1.2)\end{array}$ \\
\hline 11 & 1 & 5 & 6 & 155 & $\begin{array}{l}14.2 \\
(0.3 ; 57.8)\end{array}$ & $\begin{array}{l}96.8 \\
(92.8 ; 98.9)\end{array}$ & $\begin{array}{l}16.6 \\
(2.6 ; 59.8)\end{array}$ & $\begin{array}{l}96.2 \\
(95.0 ; 97.2)\end{array}$ & $\begin{array}{l}4.5 \\
(0.6 ; 34.0)\end{array}$ & $\begin{array}{l}0.8 \\
(0.6 ; 1.2)\end{array}$ \\
\hline 12 & 0 & 2 & 7 & 158 & $0.0(0.0 ; 40.9)$ & $98.7(95.5 ; 99.8)$ & $\begin{array}{l}0 \\
-\end{array}$ & $95.7(95.6 ; 95.8)$ & $\begin{array}{l}0.0 \\
-\end{array}$ & $1.01(1.00 ; 1.03)$ \\
\hline 13 & 2 & 22 & 5 & 138 & $28.5(3.6 ; 70.9)$ & $86.2(79.9 ; 91.1)$ & $8.3(2.5 ; 23.7)$ & $96.5(94.5 ; 97.7)$ & $2.0(0.6 ; 7.1)$ & 0.8 \\
\hline 14 & 0 & 1 & 7 & 159 & $\begin{array}{l}0.0 \\
(0.0 ; 40.9)\end{array}$ & $99.3(96.5 ; 99.9)$ & $\begin{array}{l}0 \\
-\end{array}$ & $95.7(95.7 ; 95.8)$ & $\begin{array}{l}0.0 \\
-\end{array}$ & $1.01(0,99 ; 1.02)$ \\
\hline 15 & - & - & - & - & - & - & - & - & - & - \\
\hline
\end{tabular}

\section{References}

1. a, bAthanasios G. Kaditis, Maria Luz Alonso Alvarez, An Boudewyns, Emmanouel I. Alexopoulos, et al. (2015). Obstructive sleep disordered breathing in 2-to 18-year-old children: diagnosis and management. Eur Respir J, vol. 47 (1), 69-94. doi:10.1183/13993003.00385-2015.

2. `W.M. Faizal, N.N.N. Ghazali, Irfan Anjum Badruddin, M.Z. Zainon, et al. (2019). A review of fluidstructure interaction simulation for patients with sleep related breathing disorders with obstructive sleep. Computer Methods and Programs in Biomedicine, vol. 180, 105036. doi:10.1016/j.cmpb.2019.105036.

3. ^Anna Borgström, Pia Nerfeldt, Danielle Friberg. (2013). Questionnaire OSA-18 has poor validity compared to polysomnography in pediatric obstructive sleep apnea. International Journal of Pediatric Otorhinolaryngology, vol. 77 (11), 1864-1868. doi:10.1016/j.ijporl.2013.08.030.

4. `Gili Kadmon, Sharon A. Chung, Colin M. Shapiro. (2014). I'M SLEEPY: A short pediatric sleep apnea questionnaire. International Journal of Pediatric Otorhinolaryngology, vol. 78 (12), 2116-2120. doi:10.1016/j.ijporl.2014.09.018.

5. ^Marcin Burghard, Eliza Brożek-Mądry, Antoni Krzeski. (2019). Sleep disordered breathing in children Diagnostic questionnaires, comparative analysis. International Journal of Pediatric Otorhinolaryngology, vol. 120 , 108-111. doi:10.1016/j.ijporl.2019.02.008.

6. a, bonald D Chervin, Kristen Hedger, James E Dillon, Kenneth J Pituch. (2000). Pediatric sleep questionnaire (PSQ): validity and reliability of scales for sleep-disordered breathing, snoring, sleepiness, and behavioral problems. Sleep Medicine, vol. 1 (1), 21-32. doi:10.1016/s1389-9457(99)00009-x.

7. ^Abdul Latif Hasniah, A. R. Jamalludin, A. W. Norrashidah, M. Z. Norzila, et al. (2011). Cross-cultural adaptation and reliability of pediatric sleep questionnaire in assessment of sleep-disordered breathing in the Malay speaking population. World J Pediatr, vol. 8 (1), 38-42. doi:10.1007/s12519-011-0279-3.

8. `Graziela De Luca Canto, Vandana Singh, Michael P. Major, Manisha Witmans, et al. (2014). Diagnostic 
capability of questionnaires and clinical examinations to assess sleep-disordered breathing in children. The Journal of the American Dental Association, vol. 145 (2), 165-178. doi:10.14219/jada.2013.26.

9. a, bera Panzarella, Giovanna Giuliana, Paola Spinuzza, Gaetano La Mantia, et al. (2021). Paediatric Sleep Questionnaire for Obstructive Sleep Apnoea Syndrome Screening: Is Sleep Quality Worthy of Note? Applied Sciences, vol. 11 (4), 1440. doi:10.3390/app11041440. 